\title{
MVT Regimen
}

National Cancer Institute

\section{Source}

National Cancer Institute. MVT Regimen. NCI Thesaurus. Code C10254.

A chemotherapy regimen consisting of mitoxantrone, etoposide, and thiotepa that may be used in the treatment of relapsed breast cancer. 